\title{
Crystal structure of $\left(2,2^{\prime}\right.$-bipyridine- $\left.\mathrm{K}^{2} \mathrm{~N}, \mathrm{~N}^{\prime}\right)$ bis(tri (p-tolyl)phosphine-kP)copper(I) tetrafluoroborate - 4,4'-bipyridine (2/1), $\mathrm{C}_{57} \mathrm{H}_{54} \mathrm{BCuF}_{4} \mathrm{~N}_{3} \mathrm{P}_{2}$
}

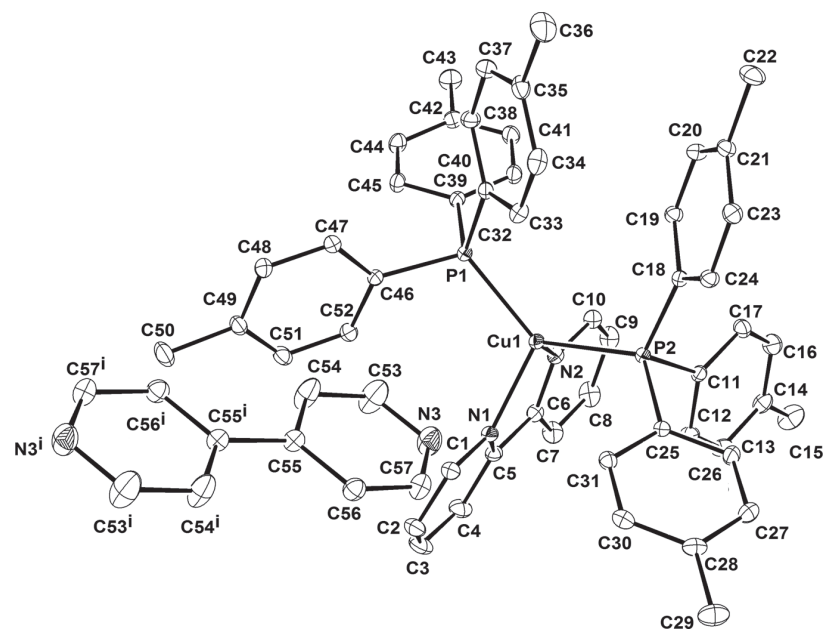

https://doi.org/10.1515/ncrs-2018-0243

Received July 15, 2018; accepted September 30, 2018; available online October 22, 2018

\section{Abstract}

$\mathrm{C}_{57} \mathrm{H}_{54} \mathrm{BCuF}_{4} \mathrm{~N}_{3} \mathrm{P}_{2}$, monoclinic, $P 2_{1} / c \quad$ (no. 14), $\quad a=$ 11.6379(12) $\AA, b=20.173(2) \AA, c=21.786(2) \AA, \beta=97.270(2)^{\circ}$, $V=5073.5(9) \AA^{3}, Z=4, R_{\mathrm{gt}}(F)=0.0440, w R_{\text {ref }}\left(F^{2}\right)=0.1283$, $T=298(2) \mathrm{K}$.

\section{CCDC no.: 1868161}

The title crystal structure is shown in the figure. Tables 1 and 2 contain details on crystal structure and measurement conditions and a list of the atoms including atomic coordinates and displacement parameters.

\section{Source of material}

The complex was obtained as an unexpected product during the synthesis of a bimetallic mixed-ligand binuclear complex including the bridging 4,4'-bipyridine ligand. Stoichiometric

\footnotetext{
*Corresponding author: Mohammed Fettouhi, Department of Chemistry, King Fahd University of Petroleum and Minerals, P.O. Box 5048, Dhahran 31261, Saudi Arabia, e-mail: fettouhi@kfupm.edu.sa Atif Fazal: Centre for Refining and Petrochemicals, Research Institute, King Fahd University of Petroleum and Minerals, Dhahran, 31261, Saudi Arabia
}

Table 1: Data collection and handling.

\begin{tabular}{ll}
\hline Crystal: & Yellow block \\
Size: & $0.45 \times 0.41 \times 0.13 \mathrm{~mm}$ \\
Wavelength: & Mo $K \alpha$ radiation $(0.71073 \AA$ A $)$ \\
$\mu:$ & $0.55 \mathrm{~mm}^{-1}$ \\
Diffractometer, scan mode: & Bruker Smart Apex, $\omega$ \\
$\theta_{\text {max }}$, completeness: & $28.3^{\circ},>99 \%$ \\
$N\left(h k l_{\text {measured }}, N(h k l)_{\text {unique }}, R_{\text {int }}:\right.$ & $68883,12618,0.034$ \\
Criterion for $I_{\text {obs }}, N(h k l)_{\mathrm{gt}}:$ & $I_{\text {obs }}>2 \sigma\left(I_{\text {obs }}\right), 9340$ \\
$N(\text { param })_{\text {refined }}:$ & 619 \\
Programs: & Bruker [1], SHELX [2, 3], ORTEP-3 \\
& {$[4]$} \\
\hline
\end{tabular}

amounts with the ratio (2/2/2/1) respectively of 2,2'-bipyridine, $\left[\mathrm{Cu}\left(\mathrm{CH}_{3} \mathrm{CN}\right)_{4}\right] \mathrm{BF}_{4}$, the phosphine and 4,4'-bipyridine were reacted in dichloromethane for $12 \mathrm{~h}$. Evaporation of the solvent yielded a yellow powder that was recrystallized from dimethylformamide, affording quality crystals of the title compound.

\section{Experimental details}

All hydrogen atoms were placed in calculated positions using a riding model with $\mathrm{C}-\mathrm{H}$ distances of $0.93 \AA$ ( $\mathrm{sp}^{2}$ carbon atoms), or $0.96 \AA$ ( $\mathrm{sp}^{3}$ carbon atoms). The isotropic displacement parameters were $U_{\text {iso }}(\mathrm{H})=1.5 U_{\text {eq }}(\mathrm{C})$ for the methyl atoms and $U_{\text {iso }}(\mathrm{H})=1.2 \mathrm{U} U_{\text {eq }}(\mathrm{C})$ for all other atoms.

Some residual density peaks near the $\mathrm{BF}_{4}{ }^{-}$ion suggest the presence of a minor disorder.

\section{Comment}

Diimine copper(I) complexes are an important and versatile class of molecules. They have found wide applications in diverse areas such as light emitting devices (LEDs), trace metal analysis, metal signaling, design of optical devices, molecular sensing and catalytic application in carbon-carbon bond formation reactions [5, 6]. Herein, we report on the crystal structure of a cocrystal containing a mixed-ligand copper(I) compound. The compound crystallizes with half uncoordinated 4,4'-bipyridine per complex formula. The copper(I) ion is bonded to the two nitrogen atoms of the chelating $2,2^{\prime}$-bipyridine ligand and the phosphorus atoms of two tri 
Table 2: Fractional atomic coordinates and isotropic or equivalent isotropic displacement parameters $\left(\AA^{2}\right)$.

\begin{tabular}{|c|c|c|c|c|c|c|c|c|c|}
\hline \multirow{2}{*}{\multicolumn{5}{|c|}{ otropic displacement parameters $\left(A^{2}\right)$}} & \\
\hline & & & & & Atom & $x$ & $y$ & $z$ & $U_{\text {iso }} * / U_{\text {eq }}$ \\
\hline Atom & $x$ & $y$ & $z$ & $U_{\text {iso }}{ }^{*} / U_{\text {eq }}$ & $\mathrm{C} 27$ & $0.1708(2)$ & $0.41782(13)$ & $0.16322(12)$ & $0.0661(6)$ \\
\hline Cu1 & $0.31019(2)$ & $0.17359(2)$ & $0.30655(2)$ & $0.04038(8)$ & $\mathrm{H} 27$ & 0.1740 & 0.4637 & 0.1595 & 0.079 * \\
\hline $\mathrm{P} 1$ & $0.40244(4)$ & $0.07880(2)$ & $0.28757(2)$ & $0.04044(12)$ & $\mathrm{C} 28$ & $0.08981(19)$ & $0.38252(14)$ & $0.12429(11)$ & $0.0591(6)$ \\
\hline $\mathrm{P} 2$ & $0.35092(4)$ & $0.27533(2)$ & $0.26976(2)$ & $0.03982(12)$ & $\mathrm{C} 29$ & $0.0057(3)$ & $0.41757(18)$ & $0.07659(14)$ & $0.0902(10)$ \\
\hline N1 & $0.13427(14)$ & $0.16312(9)$ & $0.31270(8)$ & $0.0467(4)$ & $\mathrm{H} 29 \mathrm{~A}$ & 0.0479 & 0.4416 & 0.0487 & $0.135^{*}$ \\
\hline $\mathrm{N} 2$ & $0.30615(15)$ & $0.19118(9)$ & $0.40105(8)$ & $0.0467(4)$ & $\mathrm{H} 29 \mathrm{~B}$ & -0.0407 & 0.4478 & 0.0970 & $0.135^{*}$ \\
\hline N3 & $0.0906(3)$ & $0.12847(15)$ & $0.10686(13)$ & $0.0965(8)$ & $\mathrm{H} 29 \mathrm{C}$ & -0.0436 & 0.3855 & 0.0538 & $0.135^{*}$ \\
\hline $\mathrm{C} 1$ & $0.05284(19)$ & $0.14369(12)$ & $0.26713(12)$ & $0.0583(6)$ & C30 & $0.08862(19)$ & $0.31453(13)$ & $0.13068(10)$ & $0.0558(5)$ \\
\hline $\mathrm{H} 1$ & 0.0732 & 0.1390 & 0.2274 & $0.070^{\star}$ & $\mathrm{H} 30$ & 0.0350 & 0.2898 & 0.1049 & $0.067^{*}$ \\
\hline $\mathrm{C} 2$ & $-0.0598(2)$ & $0.13043(15)$ & $0.27666(15)$ & $0.0747(7)$ & C31 & $0.16556(17)$ & $0.28195(11)$ & $0.17466(10)$ & $0.0483(5)$ \\
\hline $\mathrm{H} 2$ & -0.1142 & 0.1170 & 0.2441 & $0.090^{\star}$ & H31 & 0.1628 & 0.2360 & 0.1779 & $0.058^{*}$ \\
\hline $\mathrm{C} 3$ & $-0.0899(2)$ & $0.13743(18)$ & $0.33465(17)$ & $0.0860(9)$ & $\mathrm{C} 32$ & $0.50357(17)$ & $0.08219(9)$ & $0.23037(9)$ & $0.0428(4)$ \\
\hline $\mathrm{H} 3$ & -0.1651 & 0.1282 & 0.3423 & $0.103^{*}$ & C33 & $0.4813(2)$ & $0.12347(11)$ & $0.17944(10)$ & $0.0536(5)$ \\
\hline $\mathrm{C} 4$ & $-0.0084(2)$ & $0.15819(16)$ & $0.38192(14)$ & $0.0754(8)$ & H33 & 0.4148 & 0.1495 & 0.1746 & $0.064^{*}$ \\
\hline $\mathrm{H} 4$ & -0.0284 & 0.1641 & 0.4216 & $0.090^{\star}$ & C34 & $0.5582(3)$ & $0.12615(13)$ & $0.13556(11)$ & $0.0660(7)$ \\
\hline $\mathrm{C} 5$ & $0.10479(18)$ & $0.17038(11)$ & $0.36989(11)$ & $0.0509(5)$ & H34 & 0.5425 & 0.1544 & 0.1018 & $0.079^{*}$ \\
\hline $\mathrm{C} 6$ & $0.1983(2)$ & $0.19208(11)$ & $0.41748(10)$ & $0.0519(5)$ & $\mathrm{C} 35$ & $0.6571(2)$ & $0.08797(13)$ & $0.14077(12)$ & $0.0639(6)$ \\
\hline $\mathrm{C} 7$ & $0.1784(3)$ & $0.21398(15)$ & $0.47600(12)$ & $0.0750(8)$ & $\mathrm{C} 36$ & $0.7418(3)$ & $0.09246(19)$ & $0.09379(16)$ & $0.1024(12)$ \\
\hline $\mathrm{H} 7$ & 0.1038 & 0.2147 & 0.4870 & $0.090^{*}$ & $\mathrm{H} 36 \mathrm{~A}$ & 0.8029 & 0.1227 & 0.1084 & $0.154^{\star}$ \\
\hline $\mathrm{C} 8$ & $0.2703(3)$ & $0.23441(17)$ & $0.51687(13)$ & $0.0874(9)$ & H36B & 0.7024 & 0.1081 & 0.0551 & $0.154^{*}$ \\
\hline $\mathrm{H} 8$ & 0.2583 & 0.2495 & 0.5559 & $0.105^{\star}$ & $\mathrm{H} 36 \mathrm{C}$ & 0.7739 & 0.0494 & 0.0880 & $0.154^{*}$ \\
\hline $\mathrm{C9}$ & $0.3797(3)$ & $0.23270(14)$ & $0.50071(12)$ & $0.0742(7)$ & C37 & $0.6778(2)$ & $0.04665(14)$ & $0.19106(13)$ & $0.0662(7)$ \\
\hline H9 & 0.4429 & 0.2457 & 0.5285 & $0.089^{\star}$ & H37 & 0.7433 & 0.0198 & 0.1952 & 0.079 * \\
\hline $\mathrm{C} 10$ & $0.3943(2)$ & $0.21127(12)$ & $0.44223(10)$ & $0.0568(5)$ & $\mathrm{C} 38$ & $0.60356(19)$ & $0.04420(12)$ & $0.23552(11)$ & $0.0558(5)$ \\
\hline $\mathrm{H} 10$ & 0.4686 & 0.2107 & 0.4309 & $0.068^{\star}$ & $\mathrm{H} 38$ & 0.6208 & 0.0166 & 0.2696 & $0.067^{*}$ \\
\hline C11 & $0.36297(17)$ & $0.33243(9)$ & $0.33542(9)$ & $0.0434(4)$ & C39 & $0.49272(17)$ & $0.04555(10)$ & $0.35511(9)$ & $0.0432(4)$ \\
\hline $\mathrm{C} 12$ & $0.2613(2)$ & $0.34618(12)$ & $0.36057(11)$ & $0.0568(5)$ & $\mathrm{C} 40$ & $0.5746(2)$ & $0.08653(11)$ & $0.38746(11)$ & $0.0560(5)$ \\
\hline $\mathrm{H} 12$ & 0.1910 & 0.3305 & 0.3409 & $0.068^{\star}$ & $\mathrm{H} 40$ & 0.5809 & 0.1302 & 0.3746 & $0.067^{*}$ \\
\hline $\mathrm{C} 13$ & $0.2635(2)$ & $0.38271(14)$ & $0.41405(12)$ & $0.0660(6)$ & C41 & $0.6470(2)$ & $0.06409(13)$ & $0.43827(12)$ & $0.0620(6)$ \\
\hline $\mathrm{H} 13$ & 0.1943 & 0.3920 & 0.4295 & $0.079^{\star}$ & H41 & 0.7011 & 0.0928 & 0.4590 & $0.074^{*}$ \\
\hline C14 & $0.3661(3)$ & $0.40577(13)$ & $0.44509(12)$ & $0.0678(7)$ & $\mathrm{C} 42$ & $0.6402(2)$ & $-0.00061(12)$ & $0.45877(11)$ & $0.0579(5)$ \\
\hline C15 & $0.3665(4)$ & $0.4417(2)$ & $0.50639(15)$ & $0.1074(12)$ & C43 & $0.7186(3)$ & $-0.02565(16)$ & $0.51455(13)$ & $0.0818(8)$ \\
\hline $\mathrm{H} 15 \mathrm{~A}$ & 0.3201 & 0.4811 & 0.5002 & $0.161^{\star}$ & $\mathrm{H} 43 \mathrm{~A}$ & 0.6732 & -0.0485 & 0.5418 & $0.123^{*}$ \\
\hline H15B & 0.4445 & 0.4536 & 0.5223 & $0.161^{*}$ & $\mathrm{H} 43 \mathrm{~B}$ & 0.7576 & 0.0111 & 0.5360 & $0.123^{*}$ \\
\hline $\mathrm{H} 15 \mathrm{C}$ & 0.3351 & 0.4132 & 0.5354 & $0.161^{*}$ & $\mathrm{H} 43 \mathrm{C}$ & 0.7748 & -0.0555 & 0.5013 & $0.123^{*}$ \\
\hline C16 & $0.4664(2)$ & $0.39174(14)$ & $0.42040(13)$ & $0738(7)$ & C44 & $0.5581(2)$ & $-0.04128(12)$ & $0.42694(11)$ & $0.0629(6)$ \\
\hline $\mathrm{H} 16$ & 0.5367 & 0.4065 & 0.4408 & $0.089^{\star}$ & H44 & 0.5514 & -0.0847 & 0.4403 & $0.076^{*}$ \\
\hline C17 & $0.4657(2)$ & $0.35624(12)$ & $0.36605(12)$ & $0606(6)$ & C45 & $0.4853(2)$ & $-0.01936(11)$ & $0.37569(11)$ & $0.0558(5)$ \\
\hline $\mathrm{H} 17$ & 0.5348 & 0.3484 & 0.3501 & $0.073^{\star}$ & $\mathrm{H} 45$ & 0.4313 & -0.0482 & 0.3550 & $0.067^{*}$ \\
\hline C18 & $0.48577(16)$ & $0.28184(9)$ & $0.23616(9)$ & $0.0416(4)$ & C46 & $0.30730(16)$ & $0.00959(9)$ & $0.26085(9)$ & $0.0432(4)$ \\
\hline C19 & $0.58540(17)$ & $0.25400(11)$ & $0.26848(10)$ & $0.0485(5)$ & $\mathrm{C} 47$ & $0.32909(18)$ & $-0.03313(11)$ & $0.21393(11)$ & $0.0546(5)$ \\
\hline H19 & 0.5825 & 0.2357 & 0.3074 & $0.058^{\star}$ & $\mathrm{H} 47$ & 0.3971 & -0.0287 & 0.1960 & $0.065^{*}$ \\
\hline $\mathrm{C} 20$ & $0.68801(18)$ & $0.25346(12)$ & $0.24303(11)$ & $.0556(5)$ & C48 & $0.2503(2)$ & $-0.08254(12)$ & $0.19335(12)$ & $0.0602(6)$ \\
\hline $\mathrm{H} 20$ & 0.7540 & 0.2358 & 0.2658 & $0.067^{\star}$ & $\mathrm{H} 48$ & 0.2667 & -0.1109 & 0.1619 & $0.072^{*}$ \\
\hline $\mathrm{C} 21$ & $0.69525(18)$ & $0.27848(12)$ & $0.18449(11)$ & $0.0538(5)$ & C49 & $0.14848(19)$ & $-0.09035(12)$ & $0.21853(12)$ & $0.0597(6)$ \\
\hline $\mathrm{C} 22$ & $0.8072(2)$ & $0.27672(17)$ & $0.15651(14)$ & $0.0774(8)$ & $\mathrm{C} 50$ & $0.0597(3)$ & $-0.14192(16)$ & $0.19323(17)$ & $0.0892(10)$ \\
\hline $\mathrm{H} 22 \mathrm{~A}$ & 0.8388 & 0.3206 & 0.1562 & $0.116^{\star}$ & $\mathrm{H} 50 \mathrm{~A}$ & -0.0040 & -0.1204 & 0.1688 & $0.134^{*}$ \\
\hline $\mathrm{H} 22 \mathrm{~B}$ & 0.7927 & 0.2603 & 0.1149 & $0.116^{*}$ & $\mathrm{H} 50 \mathrm{~B}$ & 0.0321 & -0.1650 & 0.2270 & $0.134^{*}$ \\
\hline $\mathrm{H} 22 \mathrm{C}$ & 0.8613 & 0.2481 & 0.1806 & $0.116^{*}$ & $\mathrm{H} 50 \mathrm{C}$ & 0.0950 & -0.1729 & 0.1679 & $0.134^{*}$ \\
\hline $\mathrm{C} 23$ & $0.5961(2)$ & $0.30533(12)$ & $0.15245(11)$ & $0.0559(5)$ & C51 & $0.1284(2)$ & $-0.04872(13)$ & $0.26654(12)$ & $0.0632(6)$ \\
\hline $\mathrm{H} 23$ & 0.5987 & 0.3223 & 0.1130 & $0.067^{\star}$ & $\mathrm{H} 51$ & 0.0612 & -0.0539 & 0.2850 & $0.076^{*}$ \\
\hline $\mathrm{C} 24$ & $0.49330(18)$ & $0.30754(11)$ & $0.17769(10)$ & $0.0505(5)$ & $\mathrm{C} 52$ & $0.20673(19)$ & $0.00054(11)$ & $0.28754(11)$ & $0.0537(5)$ \\
\hline $\mathrm{H} 24$ & 0.4282 & 0.3265 & 0.1553 & $0.061^{*}$ & $\mathrm{H} 52$ & 0.1915 & 0.0278 & 0.3200 & $0.064^{*}$ \\
\hline $\mathrm{C} 25$ & $0.24615(16)$ & $0.31756(10)$ & $0.21361(9)$ & $0.0423(4)$ & $\mathrm{C} 53$ & $0.1228(4)$ & $0.0662(2)$ & $0.11584(17)$ & $0.1288(17)$ \\
\hline $\mathrm{C} 26$ & $0.2472(2)$ & $0.38630(11)$ & $0.20758(12)$ & $0.0580(6)$ & $\mathrm{H} 53$ & 0.1716 & 0.0562 & 0.1518 & $0.155^{*}$ \\
\hline $\mathrm{H} 26$ & 0.3000 & 0.4113 & 0.2337 & $0.070^{\star}$ & $\mathrm{C} 54$ & $0.0899(3)$ & $0.01523(16)$ & $0.07624(14)$ & $0.1021(12)$ \\
\hline
\end{tabular}

Table 2 (continued) 
Table 2 (continued)

\begin{tabular}{lrrrr}
\hline Atom & $\boldsymbol{x}$ & $\boldsymbol{y}$ & $\boldsymbol{z}$ & $\boldsymbol{U}_{\text {iso }}{ }^{*} \boldsymbol{U}_{\text {eq }}$ \\
\hline H54 & 0.1156 & -0.0276 & 0.0860 & $0.122^{*}$ \\
C55 & $0.0190(2)$ & $0.02697(12)$ & $0.02230(10)$ & $0.0539(5)$ \\
C56 & $-0.0144(2)$ & $0.09166(13)$ & $0.01201(12)$ & $0.0640(6)$ \\
H56 & -0.0621 & 0.1032 & -0.0239 & $0.077^{*}$ \\
C57 & $0.0231(3)$ & $0.13953(14)$ & $0.05529(15)$ & $0.0780(8)$ \\
H57 & -0.0015 & 0.1829 & 0.0471 & $0.094^{*}$ \\
B1 & $0.2432(3)$ & $0.7557(2)$ & $0.03899(16)$ & $0.0771(9)$ \\
F1 & $0.14482(19)$ & $0.73022(15)$ & $0.00891(10)$ & $0.1298(9)$ \\
F2 & $0.2320(3)$ & $0.82102(13)$ & $0.05247(15)$ & $0.1592(12)$ \\
F3 & $0.3280(2)$ & $0.75118(19)$ & $0.00178(13)$ & $0.1644(12)$ \\
F4 & $0.2783(2)$ & $0.72219(12)$ & $0.09161(10)$ & $0.1270(8)$ \\
\hline
\end{tabular}

( $p$-tolyl)phosphine ligands. The geometry around the metal ion is distorted tetrahedral. The 2,2'-bipyridine bite angle, $\mathrm{N} 1-\mathrm{Cu}-\mathrm{N} 2$, is $79.00(7)^{\circ}$, the $\mathrm{P}-\mathrm{Cu}-\mathrm{P}$ angle is $125.08(2)^{\circ}$ and the $\mathrm{N}-\mathrm{Cu}-\mathrm{P}$ angles are in the range $\left(103.00(5)^{\circ}-\right.$ $\left.114.89(5)^{\circ}\right)$. The $\mathrm{Cu}-\mathrm{N}$ and $\mathrm{Cu}-\mathrm{P}$ bond distances are similar to those reported for the triphenylphosphine and tricyclohexylphosphine analogous copper(I) complexes [7, 8]. Intermolecular weak, non-classical $\mathrm{CH} \cdots \mathrm{N}$ hydrogen bonding interactions take place between the uncoordinated 4, 4'-bipyridine and metal complex through 2,2'-bipyridine

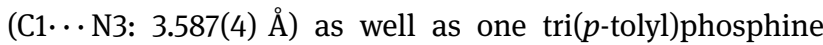

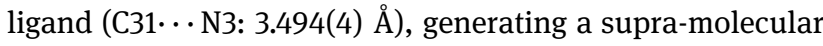
structure. Interestingly, the first $\mathrm{H}$-bonding interaction likely contributes to the non-coplanarity of the two pyridyl rings of 2,2'-bipyridine with a twisting of the pyridyl ring (N1,C1-C5) and a resulting dihedral angle of $11.74(9)^{\circ}$ between the two pyridyl rings.
Acknowledgements: The authors gratefully acknowledge the support of CRP and King Fahd University of Petroleum \& Minerals (KFUPM), Dhahran, Saudi Arabia.

\section{References}

1. BRUKER. SAINT, APEX2 and SADABS. Bruker AXS Inc., Madison, WI, USA (2009).

2. Sheldrick, G. M.: SADABS. Program for Empirical Absorption correction of Area detector Data. University of Göttingen, Germany (1996).

3. Sheldrick, G. M.: A short history of SHELX. Acta Crystallogr. A64 (2008) 112-122.

4. Farrugia, L. J.: ORTEP-3 for Windows - a version of ORTEP III with a graphical user interface (GUI). J. Appl. Crystallogr. 30 (1997) 565-565.

5. Sun, S. S.; Lees, A. J.: Transition metal based supramolecular systems: synthesis, photophysics, photochemistry and their potential applications as luminescent anion chemosensors. Coord. Chem. Rev. 230 (2002) 171-192.

6. Fazal, A.; Al-Fayez, S.; Abdel-Rahman, L. H.; Seddigi, Z. S.; AlArfaj, A.; El Ali, B.; Dastageer, M. A.; Gondal, M. A.; Fettouhi, M.: Mixed-ligand complexes of copper(I) with diimines and phosphines: effective catalysts for the coupling of phenylacetylene with halobenzene. Polyhedron 28 (2009) 4072-4076.

7. Engelhardt, L. M.; Pakawatchai, C.; White, A. H.; Healy, P. C.: Lewis-base adducts of group $1 \mathrm{~B}$ metal(I) compounds. Part 12. Structural studies of some bis(methyl-substituted pyridine)copper(I) and -silver(I) nitrates and perchlorates. J. Chem. Soc., Dalton Trans. 125 (1985) 117-123.

8. Wang, Z. W.; Cao, Q. Y.; Huang, X.; Lin, S.; Gao, X. C.: Synthesis, structure and electronic spectra of new three-coordinated copper(I) complexes with tricyclohexylphosphine and diimine ligands. Inorg. Chim. Acta 363 (2010) 15-19. 